\title{
The Second International Congress of Group Psychotherapy
}

Zurich, August 29th to 31st, 1957

Presidents

J. L. Moreno (USA) S. R. Slavson (USA) J. Bierer (Great Britain) W. C. Hulse (USA) S.

Lebovici (France) W. J. Warner (USA)

Vice-Presidents

N. Beckenstein (USA)

R. J. Corsini (USA

H. Ezriel (Great Britain)

S. H. Foulkes (Great Britain)

H. Hoff (Austria)

L. J. Hut (Netherlands)

E. E. Krapf (Argentina)

K. R. Masani (India)

F. Potts (Cuba)

T. P. Rees (Great Britain)

E. J. Rosen (Canada)

C. A. Seguin (Peru)

A. Sunier (Netherlands)

N. Waal (Norway)

Local Committee

A. Guggenbühl-Craig (Switzerland), Chairman A. Friedemann (Switzerland); H. Krebs (Switzerland)

International Committee of Group Psychotherapy

J. Bierer (Great Britain)

M. Bleuler (Switzerland)

H. Ezriel (Great Britain)

S. H. Foulkes (Great Britain)

G. Harding (Sweden)

H. Hoff (Austria)

W. C. Hulse (USA)

L. J. Hut (Netherlands)

E. Kretschmer (Germany)

E. E. Krapf (Argentina)

S. Lebovici (France)

K. R. Masani (India)

H. Meng (Switzerland)

L. Meschieri (Italy)

J. L. Moreno (USA) F. Potts (Cuba) T. P. Rees (Great Britain) P. J. Reiter (Denmark) E. J. Rosen (Canada) R. Sarro-Burbano (Spain) R. Scbindler (Austria) C. A. Seguin (Peru) S. R. Slavson 
(USA) A. Sunier (Netherlands) H. Teirich (Germany) N. Waal (Norway) W. J. Warner (USA) Contents-Inhaltsverzeichnis-Sommaire

Introduction - Geleitwort - Avant-propos

The First International Congress of Group Pyschotherapy

101

The Second International Congress of Group Psychotherapy

Addresses of Welcome - Begrüßungsansprachen - Allocutions

W. J. Warner:

Opening Address

S. Lebovící:

Allocution

108

List of Papers - Verzeichnis der Vorträge - Liste des Communications

Part I

J. Bierer (London):

Critical Analysis in Some Concepts in Present Day Group

Psychotherapy 110

R. Díatkíne (Paris):

The Place of Psychodrama in Group Psychotherapy

S. H. Foulkes (London):

Psychoanalysis, Group Psychotherapy, Group Analysis. A

Bird's Eye View of Present Trends

119

S. B. Hadden (Philadelphia, Pa.):

A Note on the Impact of Group Psychotherapy on Psychiatry 131

W. C. Hulse (New York City, N.Y.):

Einheit oder Ëinigkeit in Gruppen-Psychotherapie

J. L. Moreno (Beacon, N.Y.):

The Scientific Meaning and the Global Significance of Group

Psychotherapy 148

S. R. Slavson (New York City, N.Y.):

The Era of Group Psychotherapy

167

Z. T. Moreno (Beacon, N.Y.):

Psychodramatic Techniques

R. Schíndler (Wien):

Der soziodynamische Aspekt in der «bifokalen Gruppen-

therapie» 207

C. A. Seguin (Lima):

Psychothérapie de groupe et communication

220

B. Stokvis (Leiden):

Gruppenpsychotherapeutische Erfahrungen bei Asthmatikern 220

H. R. Teírích (Freiburg i. Br.):

Patientenklubs in der Privatpraxis. Ein Erfahrungsberícht. . 232

F. Tosquelles, R. Gentis, M. Enkin and F. Bonnet (St. Alban/Lozère):

On Group Therapy within the General Framework of Insti

tutional Therapeutics

239 